\title{
CONSUMO DE TRASTUZUMAB EN LA UNIDAD DE MEZCLAS ONCOLÓGICAS DEL HOSPITAL NACIONAL EDGARDO REBAGLIATI MARTINS, ENERO-DICIEMBRE 2009
}

\author{
Consumption of trastuzumab in the Oncological Mixtures Unit of Edgardo Rebagliati Martins \\ National Hospital, January-December 2009 \\ Jim W Gallegos ${ }^{1}$, José R Juárez ${ }^{1}$, Ada Ascarza², Mildred R Dorregaray² \\ ${ }^{1}$ Facultad de Farmacia y Bioquímica, Universidad Nacional Mayor de San Marcos. ${ }^{2}$ Hospital Nacional Edgardo Rebagliati \\ Martins-EsSALUD
}

\begin{abstract}
RESUMEN
Se estudió el consumo de trastuzumab en la Unidad de Mezclas Oncológicas (UMO) del Hospital Nacional Edgardo Rebagliati Martins (HNERM) en el 2009, mediante un análisis descriptivo-retrospectivo de las hojas de trabajo de la UMO, la utilización del Sistema de Gestión Hospitalaria (SGH) y análisis de recetas prescritas y dispensadas. Los resultados obtenidos muestran que el costo que representan los esquemas de quimioterapia fue S/. 72798,21 por paciente, siendo de mayor consumo la mezcla trastuzumab más ixabepilona con un costo promedio, para seis ciclos, de S/. 68684,52. La UMO atendió 542 prescripciones y realizó 521 preparaciones de trastuzumab las cuales generaron un gasto de S/. 3798426 y las devoluciones ascendieron a S/. 704442, lo cual generó un ahorro de $18,55 \%$.
\end{abstract}

Palabras clave: trastuzumab, Unidad de Mezclas Oncológicas (UMO), oncología médica, esquema de quimioterapia, anticuerpo monoclonal.

\section{SUMARY}

The consumption of trastuzumab in oncological mixtures Unit (UMO) of the Hospital Nacional Edgardo Rebagliati Martins was studied during 2009 through retrospective descriptive analysis of the worksheets from the UMO and using of Hospital Management System (SGH) and analysis of written and dispensed prescriptions. The results show that the cost accounting of chemotherapy schemes was $S / .72798,21$ for each patient, still the most widely consumed trastuzumab more ixabepilone mixture with an average cost for six cycles S/. 68684, 52. The UMO attended 542 prescriptions and 521 preparations with trastuzumab, which generated an expense of S/. 3798426 and refunds amounted to S/. 704442, this generated a savings of $18,55 \%$.

Keywords: trastuzumab, Mixtures Oncologic Unit (UMO), medical oncology, chemotherapy regimen, monoclonal antibody.

\section{INTRODUCCIÓN}

Trastuzumab es un anticuerpo monoclonal derivado del ADN recombinante humanizado que se une selectivamente y, con alta afinidad para el dominio extracelular, al receptor 2 del factor de crecimiento epidérmico (HER2) ${ }^{(1,2)}$. Fue aprobado por la FDA para el tratamiento del cáncer de mama metastásico, cuyo(s) tumor(es) sobreexprese(n) la proteína HER2, en aquellos pacientes que han recibido uno o más regímenes de quimioterapia, así como, en cáncer de mama temprano que exprese HER2 ${ }^{(3)}$. El gen HER2 (también conocido como HER2/neu y el gen ErbB2) es amplificado en un $20-30 \%$ en la primera etapa de la sobreexpresión del cáncer de mama ${ }^{(4)}$.

Trastuzumab es sinérgico con otros tipos de agentes citotóxicos, pero sólo en aquellos casos que sobrexpresen HER2. En otros tipos de cáncer, como en tumores sólidos, de los cuales han sido reportados: cáncer colo-rectal y de pulmón de células pequeñas ${ }^{(5)}$, trastuzumab se une al dominio IV del segmento extracelular del receptor HER2/neu. Células tratadas con trastuzumab al someterse a la detención durante la fase Gi del ciclo celular han reducido su proliferación.

Como se muestra en la parte A de la figura 1, los cuatro miembros de la familia HER son: HER1, HER2, HER$_{3}$ y HER 4 . Hay receptores ligando-específicos para HER1, HER3 y HER4. Una tirosina quinasa de dominio intracelular existe para HER1, HER2 y HER4. La unión de trastuzumab a un dominio yuxtamembranoso de HER2 reduce la pérdida del dominio extracelular, reduciendo así el p95 (Figura 1 C). Trastuzumab puede reducir la señalización de HER2 físicamente, ya sea inhibiendo la homodimerización o la heterodimerización (Figura 1D); puede reunir las células inmunes efectoras Fc-competentes y los demás componentes de la 


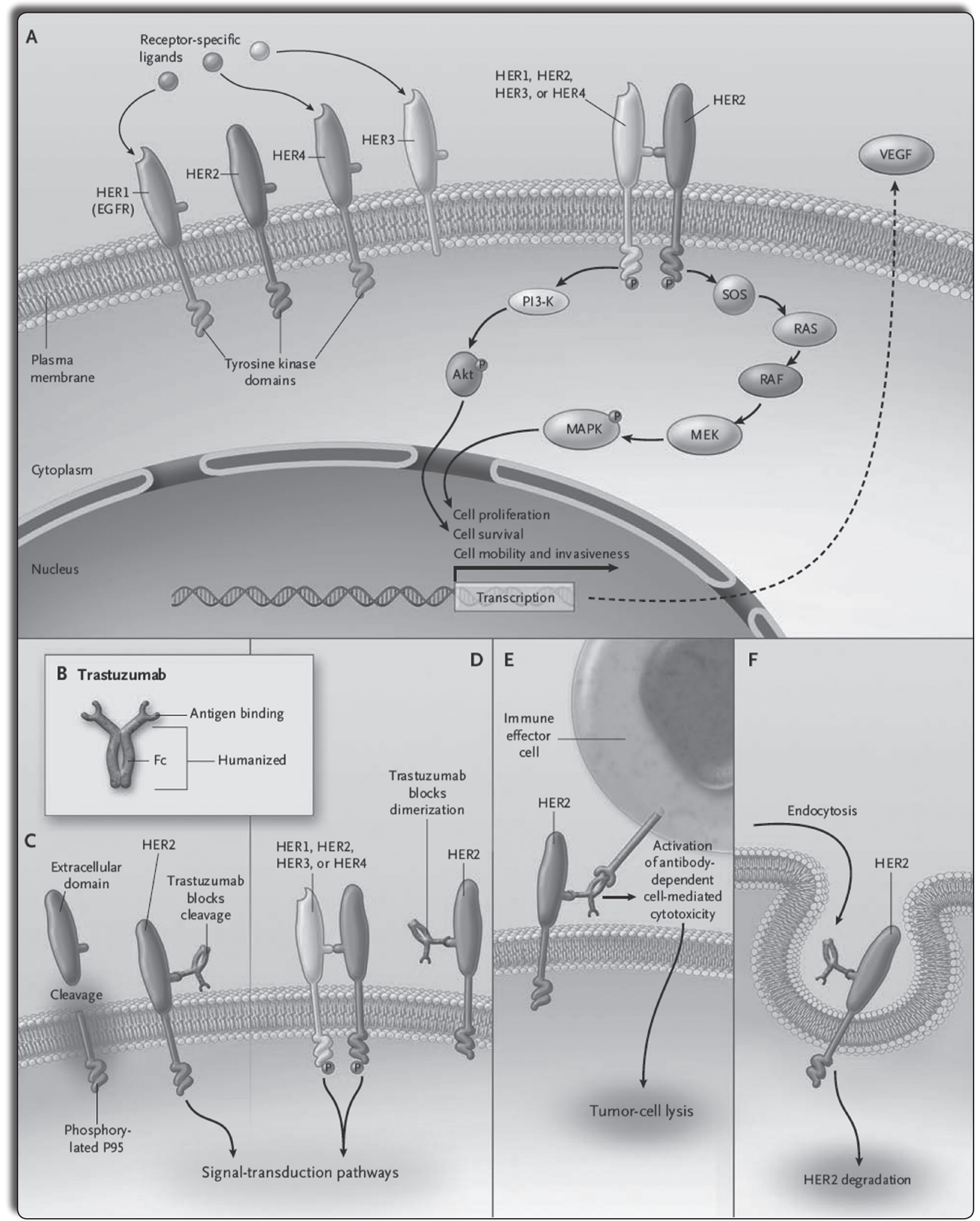

Figura 1. $\boldsymbol{A}$. Transducción de Señales por la familia HER. $\boldsymbol{B}, \boldsymbol{C}, \boldsymbol{D}, \boldsymbol{E} \boldsymbol{y} \boldsymbol{F}$. Posibles mecanismo de acción para trastuzumab. Fuente: The New England Journal of Medicine 2007; 357:39-51. 
citotoxicidad mediada por células dependientes de anticuerpos, lo que conduce a la muerte decélulas tumorales (Figura $1 \mathrm{E}$ ). Se han postulado mecanismos adicionales, entre otros "down-regulatión" de los receptores a través de la endocitosis (Figura $\mathrm{1F})^{(6)}$.

En general, la terapia con trastuzumab es bien tolerada; los eventos adversos más comunes están relacionados con la infusión, principalmente fiebre $y$ escalofríos, que ocurren en aproximadamente en el $40 \%$ de los pacientes durante la perfusión inicial $(7,8)$. Una de las complicaciones significativas de trastuzumab es su efecto sobre el corazón, que está asociado con disfunción cardíaca en el 2-7\% de los $\operatorname{casos}^{(9)}$.

\section{Aproximadamente}

el $10 \%$ de los pacientes no pueden tolerar esta droga, debido a problemas preexistentes en el corazón; por lo que los médicos deben evaluar el riesgo de cáncer recurrente contra un mayor riesgo de muerte por enfermedad cardíaca en esta población. El riesgo de cardiopatía se incrementa cuando trastuzumab es combinado con antraciclinas, que a su vez están asociadas con toxicidad cardiaca ${ }^{(10)}$.

Se realizó una búsqueda de revisiones sistemáticas publicadas en Pubmed, Cocrhane y Tripdatabase, encontrándose un estudio de costo-efectividad de trastuzumab en cáncer de mama temprano, el cual compara el tratamiento con
Tabla 1. Esquemas de quimioterapia que incluyen trastuzumab en el servicio de Oncología médica

\begin{tabular}{|c|c|c|c|}
\hline № Esquema & Esquema terapéutico & Frecuencia del ciclo & Diagnostico \\
\hline 1 & $\begin{array}{l}\text { Trastuzumab 4mg/kg durante } \\
90 \text { minutos (Dosis de carga) } \\
\text { Trastuzumab } 2 \mathrm{mg} / \mathrm{kg} \\
\text { durante } 30 \text { minutos (Dosis de } \\
\text { mantenimiento) }\end{array}$ & $\begin{array}{l}\text { Día } 1 \\
\text { Día } 8 \text { y } 15 \\
\text { Repetir cada } 21 \text { días }\end{array}$ & $\begin{array}{c}\text { Cáncer de mama } \\
\text { metastásico HER2 +++ }\end{array}$ \\
\hline 2 & $\begin{array}{l}\text { Trastuzumab 4mg/kg durante } \\
90 \text { minutos (Dosis de carga) } \\
\text { Paclitaxel } 175 \mathrm{mg} / \mathrm{kg} \text { durante } \\
180 \text { minutos } \\
\text { Trastuzumab } 2 \mathrm{mg} / \mathrm{kg} \\
\text { durante } 30 \text { minutos (Dosis de } \\
\text { mantenimiento }\end{array}$ & $\begin{array}{l}\text { Día } 1 \\
\text { Día } 1 \\
\text { Día } 8 \text { y } 15 \\
\text { Repetir cada } 21 \text { días }\end{array}$ & $\begin{array}{c}\text { Cáncer de mama } \\
\text { metastásico HER2 +++ }\end{array}$ \\
\hline 3 & $\begin{array}{l}\text { Trastuzumab } 4 \mathrm{mg} / \mathrm{kg} \text { durante } \\
90 \text { minutos (Dosis de carga) } \\
\text { Docetaxel } 100 \mathrm{mg} / \mathrm{kg} \text { IV } \\
\text { Trastuzumab } 2 \mathrm{mg} / \mathrm{kg} \\
\text { durante } 30 \mathrm{minutos} \text { (Dosis de } \\
\text { mantenimiento }\end{array}$ & $\begin{array}{l}\text { Día } 1 \\
\text { Día } 1 \\
\text { Día } 8 \text { y } 15 \\
\text { Repetir cada } 21 \text { días }\end{array}$ & $\begin{array}{c}\text { Cáncer de mama } \\
\text { metastásico HER2 +++ }\end{array}$ \\
\hline 4 & $\begin{array}{l}\text { Trastuzumab } 4 \mathrm{mg} / \mathrm{kg} \text { durante } \\
90 \text { minutos (Dosis de carga) } \\
\text { Gemcitabina } 100 \mathrm{mg} / \mathrm{m}^{2} \mathrm{IV} \\
\text { Trastuzumab } 2 \mathrm{mg} / \mathrm{kg} \\
\text { durante } 30 \mathrm{minutos} \text { (Dosis de } \\
\text { mantenimiento }\end{array}$ & $\begin{array}{l}\text { Día } 1 \\
\text { Día } 8 \text { y } 15 \\
\text { Semanal después de la } \\
\text { primera dosis. } \\
\text { Repetir cada } 28 \text { días }\end{array}$ & $\begin{array}{c}\text { Cáncer de mama } \\
\text { metastásico HER2 +++ }\end{array}$ \\
\hline 5 & $\begin{array}{l}\text { Trastuzumab } 8 \mathrm{mg} / \mathrm{kg} \text { durante } \\
90 \text { minutos (Dosis de carga) } \\
\text { Ixabepilona } 40 \mathrm{mg} / \mathrm{m} 2 \text { IV } \\
\text { Trastuzumab } 6 \mathrm{mg} / \mathrm{kg} \\
\text { durante } 30 \mathrm{minutos} \text { (Dosis de } \\
\text { mantenimiento }\end{array}$ & $\begin{array}{l}\text { Día } 1 \\
\text { Día } 1 \\
\text { Día } 8 \text { y } 15 \\
\text { Repetir cada } 21 \text { días }\end{array}$ & $\begin{array}{c}\text { Cáncer de mama } \\
\text { metastásico HER2 +++ }\end{array}$ \\
\hline 6 & $\begin{array}{l}\text { Trastuzumab } 4 \mathrm{mg} / \mathrm{kg} \text { durante } \\
90 \text { minutos (Dosis de carga) } \\
\text { Vinorelbina } 25 \mathrm{mg} / \mathrm{m}^{2} \text { durante } 5 \\
\text { a } 10 \text { minutos. } \\
\text { Trastuzumab } 2 \mathrm{mg} / \mathrm{kg} \\
\text { durante } 30 \text { minutos (Dosis de } \\
\text { mantenimiento }\end{array}$ & $\begin{array}{l}\text { Día } 1 \\
\text { Día } 1 \text { y semanal por } 8 \\
\text { semanas. } \\
\text { Día } 8 \text { y semanal por } 8 \\
\text { semanas. }\end{array}$ & $\begin{array}{c}\text { Cáncer de mama } \\
\text { metastásico HER2 +++ }\end{array}$ \\
\hline 7 & $\begin{array}{l}\text { Trastuzumab } 4 \mathrm{mg} / \mathrm{kg} \text { durante } \\
90 \text { minutos (Dosis de carga) } \\
\text { Vinorelbina } 25 \mathrm{mg} / \mathrm{m}^{2} \text { durante } 5 \\
\text { a } 10 \text { minutos. } \\
\text { Gemcitabina } 800 \mathrm{mg} / \mathrm{m}^{2} \text { durante } \\
30 \text { minutos } \\
\text { Trastuzumab } 2 \mathrm{mg} / \mathrm{kg} \\
\text { durante } 30 \text { minutos (Dosis de } \\
\text { mantenimiento }\end{array}$ & $\begin{array}{l}\text { Día } 1 \\
\text { Día } 1 \text { y } 8 \\
\text { Día } 1 \text { y repetir cada } 21 \\
\text { días. } \\
\text { Día } 8 \text { y semanal por } 8 \\
\text { semanas. }\end{array}$ & $\begin{array}{c}\text { Cáncer de mama } \\
\text { metastásico HER2 +++ }\end{array}$ \\
\hline 8 & $\begin{array}{l}\text { Trastuzumab 4mg/kg durante } \\
90 \text { minutos (Dosis de carga) } \\
\text { Docetaxel } 75 \mathrm{mg} / \mathrm{m} 2 \mathrm{IV} \\
\text { Carboplatino } 6 \mathrm{AUC} \text { IV } \\
\text { Trastuzumab } 2 \mathrm{mg} / \mathrm{kg} \\
\text { durante } 30 \mathrm{minutos} \text { (Dosis de } \\
\text { mantenimiento }\end{array}$ & $\begin{array}{l}\text { Día } 1 \\
\text { Día } 1 \\
\text { Día } 1 \\
\text { Días } 8 \text { y 15, repetir cada } \\
21 \text { días. }\end{array}$ & $\begin{array}{l}\text { Cáncer de mama } \\
\text { metastásico HER2 +++ }\end{array}$ \\
\hline 9 & $\begin{array}{l}\text { Trastuzumab 4mg/kg durante } \\
90 \text { minutos (Dosis de carga) } \\
\text { Paclitaxel } 175 \mathrm{mg} / \mathrm{m}^{2} \\
\text { Carboplatino } 6 \mathrm{AUC} \text { IV } \\
\text { Trastuzumab } 2 \mathrm{mg} / \mathrm{kg} \\
\text { durante } 30 \mathrm{minutos} \text { (Dosis de } \\
\text { mantenimiento }\end{array}$ & $\begin{array}{l}\text { Día } 1 \\
\text { Día } 1 \\
\text { Día } 1 \\
\text { Días } 8 \text { y 15, repetir cada } \\
21 \text { días. }\end{array}$ & $\begin{array}{c}\text { Cáncer de mama } \\
\text { metastásico HER2 +++ }\end{array}$ \\
\hline 10 & $\begin{array}{l}\text { Trastuzumab } 4 \mathrm{mg} / \mathrm{kg} \text { durante } \\
90 \text { minutos (Dosis de carga) } \\
\text { Ciclofosfamida } 600 \mathrm{mg} / \mathrm{m}^{2} \mathrm{IV} \\
\text { Doxorrubicina } 60 \mathrm{mg} / \mathrm{m}^{2} \mathrm{IV} \\
\text { Trastuzumab } 2 \mathrm{mg} / \mathrm{kg} \\
\text { durante } 30 \mathrm{minutos} \text { (Dosis de } \\
\text { mantenimiento }\end{array}$ & $\begin{array}{l}\text { Día } 1 \\
\text { Día } 1 \\
\text { Día } 1 \\
\text { Días } 8 \text { y } 15 \text {, repetir cada } \\
21 \text { días. } \\
\end{array}$ & $\begin{array}{c}\text { Cáncer de mama } \\
\text { metastásico HER2 +++ }\end{array}$ \\
\hline
\end{tabular}


antraciclinas $(\mathrm{N})$ sin trastuzumab $(\mathrm{T})$, antraciclinas (N) con trastuzumab adyuvante (AT) y no antraciclinas (NA) con trastuzumab (T); concluyéndose que hay un incremento en el costo ICEI (costo efectividad por año de vida ganado) de antraciclinas con trastuzumab (doxorrubicina + ciclofosfamida + paclitaxel + trastuzumab) siendo menos costoso que el tratamiento de no antraciclinas con trastuzumab (docetaxel + carboplatino+trastuzumab). Losautoressugieren realizar estudios acerca de la toxicidad cardíaca producida, como reacción adversa, por la combinación de antraciclinas y trastuzumab ya que no hay estudios previos ${ }^{(11)}$.

En otro estudio costo-efectividad para cáncer de mama temprano, se evaluaron dos regímenes de tratamiento; uno fue el Proyecto nacional de cirugía adyuvante de mama e intestino (NSABP B 31 por sus siglas en inglés) y el Grupo de tratamiento para cáncer central del norte(NCCTG N9831 por sus siglas en inglés). Estos concluyeron que la adición de trastuzumab como terapia adyuvante reduce el riesgo de recurrencia e incrementa la sobrevida de los pacientes además de ser bien aceptada en los tratamientos oncológicos ${ }^{(12)}$.

En el Perú, aun no hay estudios de consumo referentes a trastuzumab, solo se adquiere en EsSalud, por aprobación del Comité farmacológico, para el servicio de Oncología médica desde el año 2008 y, su reconstitución, preparación y estabilidad se encarga a la Unidad de Mezclas Oncológica s (UMO) del Hospital Nacional Edgardo Rebagliati Martins (HNERM).

El objetivo de la presente investigación fue analizar el consumo de trastuzumab en la Unidad de Mezclas Oncológicas del HNERM de enero-diciembre 2009.

\section{MATERIALES Y MÉTODOS}

\section{Materiales}

- Hojas de trabajo donde se detalla el servicio, nombre del preparado, dosis y dilución de cada mes(enero-diciembre 2009).

- Sistema de gestión hospitalaria (SGH).

- Reporte de devolución mensual a la UMO (enero-diciembre 2009).

\section{Método}

El presente, es un análisis retrospectivo-descriptivo para evaluar el consumo de trastuzumab en la UMO del HNERM durante el
Tabla 2. Pacientes que reciben su terapia con trastuzumab en los diferentes esquemas de quimioterapia, durante el periodo de estudio.

\begin{tabular}{cccc}
\hline $\begin{array}{c}\text { Número de } \\
\text { esquema }\end{array}$ & $\begin{array}{c}\text { Esquema } \\
\text { terapéutico }\end{array}$ & $\begin{array}{c}\text { Cantidad de } \\
\text { pacientes }\end{array}$ & \% \\
\hline 1 & TRZ & 55 & 61,8 \\
2 & TRZ+PCL & 12 & 13,5 \\
3 & TRZ+DOCEX & 7 & 7,9 \\
4 & TRZ+GEM & 2 & 2,2 \\
5 & TRZ+IXAB & 1 & 1,1 \\
6 & TRZ+NLV & 3 & 3,4 \\
7 & TRZ+NLV+GEM & 5 & 5,6 \\
8 & TRZ+DOCEX+CBDCA & 2 & 2,2 \\
9 & TRZ+PCL+CBDCA & 1 & 1,1 \\
10 & TRX+DOXO+CTX & 1 & 1,1 \\
& TOTAL & $\mathbf{8 9}$ & $\mathbf{1 0 0 , 0}$ \\
\hline
\end{tabular}

Tabla 3. Costo que representa trastuzumab en cada esquema de quimioterapia, basado en una preparación para el paciente

\begin{tabular}{ccc}
\hline $\begin{array}{c}\text { Número de } \\
\text { esquema }\end{array}$ & $\begin{array}{c}\text { Esquema } \\
\text { terapéutico }\end{array}$ & $\begin{array}{c}\text { Costo en } \\
\text { Nuevos Soles (S/.) }\end{array}$ \\
\hline 1 & TRZ (MONO) & 6583,57 \\
2 & TRZ+PCL & 6624,31 \\
3 & TRZ+DOCEX & 6759,27 \\
4 & TRZ+GEM & 6764,64 \\
5 & TRZ+IXABEP & 11447,42 \\
6 & TRZ+NVL & 7141,17 \\
7 & TRZ+GEM+NVL & 7322,24 \\
8 & TRZ+DOCEX+CBDCA & 6833,66 \\
9 & TRZ+CBDCA+PCL & 6698,70 \\
10 & TRZ+DOXO+CTX & 6623,23 \\
& TOTAL & $\mathbf{7 2 7 9 8 , 2 1}$ \\
\hline
\end{tabular}

periodo enero-diciembre 2009. En el se que cuantifica el número de pacientes por esquema terapéutico, se analiza el diagnóstico, dosis y costo, e investiga la cantidad y frecuencia con que los pacientes reciben su terapia.

\section{RESULTADOS Y DISCUSIÓN}

Para la identificación de los esquemas de tratamiento en los que se utiliza trastuzumab, en la tabla 1 se observa que, durante el periodo de estudio, 
Tabla 4. Costo mínimo y máximo por ciclo de tratamiento según esquema terapéutico y diagnostico.

\begin{tabular}{|c|c|c|c|c|c|c|c|}
\hline $\begin{array}{l}\text { Número de } \\
\text { esquema }\end{array}$ & $\begin{array}{c}\text { Esquema } \\
\text { terapéutico }\end{array}$ & Diagnostico & Dosis/mg & Costo mínimo & Costo máximo & $\begin{array}{c}\text { Mínimo en } 6 \\
\text { ciclos }\end{array}$ & $\begin{array}{c}\text { Máximo en } 6 \\
\text { ciclos }\end{array}$ \\
\hline 1 & Trastuzumab & Ca. Mama HER2 +++ & $90-648$ & 6583,57 & 13167,14 & 39501,42 & 79002,84 \\
\hline 2 & $\begin{array}{c}\text { Trastuzumab } \\
\text { Paclitaxel }\end{array}$ & Ca. Mama HER2 +++ & $90-440$ & 6624,31 & 6665,05 & 39745,86 & 39990,3 \\
\hline 3 & $\begin{array}{c}\text { Trastuzumab } \\
\text { Docetaxel }\end{array}$ & Ca. Mama HER2 +++ & $130-648$ & 6759,37 & 13518,54 & 40556,22 & 81111,24 \\
\hline 4 & $\begin{array}{l}\text { Trastuzumab } \\
\text { Gemcitabina }\end{array}$ & Ca. Mama HER2 +++ & 640 & 13348,21 & 13529,28 & 80089,26 & 81175,68 \\
\hline 5 & $\begin{array}{l}\text { Trastuzumab } \\
\text { Ixabepilona }\end{array}$ & Ca. Mama HER2 +++ & 440 & 11447,42 & 11447,42 & 68684,52 & 68684,52 \\
\hline 6 & $\begin{array}{l}\text { Trastuzumab } \\
\text { Vinorelbina }\end{array}$ & Ca. Mama HER2 +++ & $110-375$ & 7141,17 & 7698,77 & 42847,02 & 46192,62 \\
\hline 7 & $\begin{array}{l}\text { Trastuzumab } \\
\text { Gemcitabina } \\
\text { Vinorelbina }\end{array}$ & Ca. Mama HER2 +++ & $120-440$ & 7322,24 & 8060,91 & 43933,44 & 48365,46 \\
\hline 8 & $\begin{array}{c}\text { Trastuzumab } \\
\text { Carboplatino } \\
\text { Docetaxel }\end{array}$ & Ca. Mama HER2 +++ & $300-440$ & 6833,56 & 7113,75 & 41001,36 & 42682,5 \\
\hline 9 & $\begin{array}{c}\text { Trastuzumab } \\
\text { Carboplatino } \\
\text { Paclitaxel }\end{array}$ & Ca. Mama HER2 +++ & 490 & 13282,27 & 13397,4 & 79693,62 & 80384,4 \\
\hline 10 & $\begin{array}{l}\text { Trastuzumab } \\
\text { Doxorrubicina } \\
\text { Ciclofosfamida }\end{array}$ & Ca. Mama HER2 +++ & 440 & 6623,23 & 6669,66 & 39739,38 & 40017,96 \\
\hline
\end{tabular}

Tabla 5. Costo mínimo y máximo por ciclo de tratamiento según esquema terapéutico y diagnostico.

\begin{tabular}{|c|c|c|c|c|c|c|}
\hline Mes & $\begin{array}{l}\text { Número de } \\
\text { ampollas } \\
\text { atendidas }\end{array}$ & $\begin{array}{l}\text { Número de } \\
\text { ampollas } \\
\text { devueltas }\end{array}$ & $\begin{array}{c}\text { Número de } \\
\text { preparaciones }\end{array}$ & $\begin{array}{c}\text { Precio de } \\
\text { ampollas (S/.) }\end{array}$ & $\begin{array}{c}\text { Precio de } \\
\text { devolución (S/.) }\end{array}$ & $\begin{array}{c}\text { Ahorro en } \\
\text { devoluciones (\%) }\end{array}$ \\
\hline Enero & 31 & 6 & 28 & 203877 & 39501 & 19,38 \\
\hline Febrero & 41 & 6 & 34 & 269762 & 39501 & 14,64 \\
\hline Marzo & 44 & 6 & 37 & 289579 & 39501 & 13,64 \\
\hline Abril & 42 & 9 & 36 & 276444 & 59252 & 21,43 \\
\hline Mayo & 37 & 3 & 35 & 243555 & 19751 & 8,11 \\
\hline Junio & 47 & 3 & 40 & 309425 & 19751 & 6,38 \\
\hline Julio & 44 & 26 & 41 & 289695 & 171173 & 59,09 \\
\hline Agosto & 53 & 12 & 51 & 348968 & 79003 & 22,64 \\
\hline Septiembre & 61 & 3 & 55 & 401648 & 19751 & 4,92 \\
\hline Octubre & 59 & 7 & 58 & 388485 & 46085 & 11,86 \\
\hline Noviembre & 67 & 8 & 58 & 441168 & 52669 & 11,94 \\
\hline Diciembre & 51 & 18 & 48 & 335818 & 118504 & 35,29 \\
\hline Total & 577 & 107 & 521 & 3798426 & 704442 & 18,55 \\
\hline
\end{tabular}

se hicieron uso de diez esquemas diferentes de quimioterapia en los cuales se incluyó este principio activo. Dentro de estos esquemas se puede apreciar lo siguiente:

- Todos están indicados para los pacientes que tienen cáncer de mama metastásico HER2 +++.

- No se identificó otro diagnóstico que requiera la utilización y preparación de trastuzumab.
- La dosis de trastuzumab prescrita para los esquemas de quimioterapia van de 2 a $8 \mathrm{mg} /$ $\mathrm{kg}$ de peso, dependiente del tipo de esquema utilizado para el paciente y los fármacos asociados (medicamentos oncológicos).

- El tiempo de administración de trastuzumab no excedió de 90 minutos, ya que esto puede provocar reacciones adversas, tales como: taquicardia, 
hipotensión y desvanecimiento del paciente.

En la cuantificación del número de pacientes por esquema terapéutico, donde se usó trastuzumab, se encontraron los siguientes resultados:

- Fueron 89 pacientes mujeres.

- A todas las pacientes que recibieron trastuzumab se les infundió su terapia por consulta externa y no necesitaron de hospitalización.

En la tabla 2 se puede identificar el número de pacientes por esquema terapéutico, donde de los diez esquemas utilizados el $61,8 \%$ correspondió a quienes recibieron trastuzumab como monoterapia, esto debido a que la mayoría de pacientes tienen un máximo de 6 meses de terapia y, según los esquemas de tratamiento con trastuzumab aplicados en el Servicio de Oncología Médica, el inicio del tratamiento con trastuzumab como monoterapia es de $4 \mathrm{mg} / \mathrm{kg}$ como dosis de carga y luego $2 \mathrm{mg} / \mathrm{kg}$ como dosis de mantenimiento, en la mayoría de esquemas.

En la distribución de pacientes por edad, se evidenció que el 43,8\% estaban entre 41 a 50 años; esto se puede deber a que las pacientes ya han recibido un tratamiento anterior con quimioterapia y han avanzado en su enfermedad expresando el gen HER2, lo que las convierte en elegibles al tratamiento con trastuzumab o también a que la prevalencia de la enfermedad y del diagnóstico estaban dentro del rango encontrado durante el periodo de estudio.

En relación al costo, en nuevos soles, de los esquemas evaluados para cada paciente, se observa que el esquema 5 (trastuzumab + ixabepilona) es el más alto, debido al costo de ixabepilona, que pertenece a las epotilonas, un nuevo grupo de fármacos para el cáncer de mama metastásico. Para nuestro caso sólo una paciente recibió este esquema porque su utilización en Oncología Médica es reciente y su costo es superior al de trastuzumab (tabla 3 ).

En la tabla 4, se observa el costo al cumplir 6 ciclos de quimioterapia, tanto en mínimo y máximo (cumplir veintiuno o veintiocho días de tratamiento y volver a empezar); el esquema terapéutico 4 (trastuzumab + gemcitabina) es el esquema que más costo reportó durante el periodo de estudio, debido a que la dosis que se utiliza para ese esquema $(640 \mathrm{mg} / \mathrm{kg})$ equivalen a 2 unidades de trastuzumab. Cabe mencionar que los costos obtenidos son por unidad de trastuzumab y no por los miligramos utilizados en la dosis del esquema.

Los resultados de las devoluciones de trastuzumab y el costo que representa su utilización durante el periodo de estudio se muestran en la tabla 5, así como el número de preparados quimioterápicos de trastuzumab elaborados por parte de la UMO y la cantidad de remanentes que se obtuvieron en cada esquema terapéutico; observándose que el esquema 1 (trastuzumab monoterapia) es el de mayor demanda y consecuentemente el esquema que generó mayor cantidad de remanentes. Esto debido a que la dosis usada en dicho esquema es muy variable (9o hasta $648 \mathrm{mg} / \mathrm{kg}$ ) y, debido a que la unidad de trastuzumab contiene $440 \mathrm{mg}$, que sólo pueden utilizarse durante el día o refrigerarse hasta el día siguiente.

En la tabla 5 también se indica el costo (en nuevos soles) de las ampollas utilizadas por la UMO y los remanentes de que se devolvieron a la farmacia citotóxicos. Como se puede apreciar, de 577 ampollas, 107 fueron devueltas, lo que equivale al $18,55 \%$. De igual manera, el costo de las devoluciones de trastuzumab ascendió a 704442 nuevos soles, esto implica que la UMO genera un ahorro considerable con respecto a este medicamento, tanto en lo referente a su reconstitución como a su preparación (Figura 2), por lo cual se considera a la UMO un eje en rentabilidad para el Departamento de Farmacia de la Red Asistencial Rebagliati.

\section{CONCLUSIONES}

En el Servicio de Oncología Médica, respecto al tratamiento de cáncer de mama metastásico que expresa el gen HER2, existen diez esquemas terapéuticos que incluyen trastuzumab; del $100 \%$ de las pacientes, el 36\% tenían un tiempo de tratamiento de 1 a 3 meses y el $48,3 \%$ estaban entre los 41 a 50 años de edad.

De los diez esquemas terapéuticos, el esquema 1 (trastuzumab monoterapia) lo recibieron en $61,8 \%$ de las pacientes y el gasto real que generó su consumo durante el periodo de estudio fue de 3798719,89 nuevos soles; a la vez se originó un ahorro de 704442 nuevos soles, lo que corresponde al 18,55\%. Asimismo, la cantidad de unidades de trastuzumab utilizadas por la UMO fue de 577, generando 107 remanentes, lo que equivale al $18,55 \%$.

\section{REFERENCIAS BIBLIOGRÁFICAS}

1. Coussens L, Yang-Feng TL, Liao Y-C, Chen E, Gray A, McGrath J et al. Tyrosine kinase receptor with extensive homology to EGF receptor shares chromosomal location with neu oncogene. Science 1985; 230 (1): 1132-9.

2. Press MF, Pike MC, Chazin VR, Hung G, Udove JA, 
Markowicz M et al. Her-2/neu expression in nodenegative breast cancer: direct tissue quantitation by computerized image analysis and association of overexpression with increased risk of recurrent disease. Cancer Res 1993; 53: 4960-70.

3. FDA. Trastuzumab product approval information - Licensing action 9/25/98. [En línea] Acceso 03 Marzo 2010. Disponible en: http://www.fda.gov/ downloads/Drugs/DevelopmentApprovalProcess/

HowDrugsareDevelopedandApproved/ ApprovalApplications/ TherapeuticBiologicApplications/ucmo9136o.pdf.

4. Bange J, Zwick E, Ullrich A. (2001). Molecular targets for breast cancer therapy and prevention. Nature Medicine 2001; 7 (5): 548-52.

5. Brunton L, Parker K, Blumenthal D, Buxton I.Eds. Goodman\&Gilman. Las Bases farmacológicas de la terapéutica. $11^{\mathrm{a}}$ ed. McGraw-Hill Interamericana. México DF, 2008. p. 901-4.

6. Hudis CA. Trastuzumab - Mechanism of action and use in clinical practice. N Engl J Med 2007; 357: 39-51.

7. Tokuda Y, Watanabe T, Omura Y et al. Dose escalation and pharmacokinetic study of humanized anti-HER2 monoclonal antibody in patients with $\mathrm{HER} 2 / n e u-$ overexpressing metastatic breast cancer. $\mathrm{Br}$ J Cancer 1999; 81: 1419-25.

8. Perry CM, Wiseman LR. Trastuzumab. BioDrugs. 1999; 12(2):129-35.
9. Seidman A et al. Cardiac Dysfunction in the trastuzumab clinical trials experience. J Clin Oncol 200220 (5): 121521. [En línea] Acceso 16 Marzo 2010. Disponible en http:// jco.ascopubs.org/cgi/content/full/20/5/1215.

10. Rothenberg MJ, Ling V. Multidrug resistance: molecular biology and clinical relevance. J Natl Cancer lnst 1989; 81: 907-10.

11. Kurian A, Thompson R, Gaw A, Arai S, Ortiz R, Garber A. A cost-effectiveness analysis of adjuvant trastuzumab regimens in early HER2/neu-positive breast cancer. J Clin Oncol 2007; 25(6): 634-41. [En línea] Acceso 10 Marzo 2010. Disponible en http://jco.ascopubs.org/cgi/content/ full/25/6/634.

12. Garrison L, Lubeck D, Lalla D, Paton V, Dueck A, Pérez E. Cost-Effectiveness Analysis of trastuzumab in the adjuvant setting for treatment of HER2-positive breast cancer. Cancer 2007; 110(3): 489-98.

Manuscrito recibido el: 17/10/2011

Aceptado para su publicación el: 22/11/2011

\section{Correspondencia:}

Nombre: Dr. José Juárez Eyzaguirre

Dirección: Jr. Los Tulipanes 343 - Lima 12 /

e-mail:_ jjuareze@unmsm.edu.pe 